\title{
COMMUTING POLYNOMIAL OPERATIONS OF DISTRIBUTIVE LATTICES
}

\author{
MIKE BEHRISCH, MIGUEL COUCEIRO, KEITH A. KEARNES, ERKKO LEHTONEN, \\ AND ÁGNES SZENDREI
}

\begin{abstract}
We describe which pairs of distributive lattice polynomial operations commute.
\end{abstract}

\section{INTRODUCTION}

Let $f: X^{m} \rightarrow X$ and $g: X^{n} \rightarrow X$ be operations on $X$ and let $\left[x_{i j}\right] \in X^{m \times n}$ be a matrix of elements of $X$. By $f \circ g\left(\left[x_{i j}\right]\right)$ we mean the value obtained by first applying $g$ to the rows of $\left[x_{i j}\right]$ and then applying $f$ to the column of results.

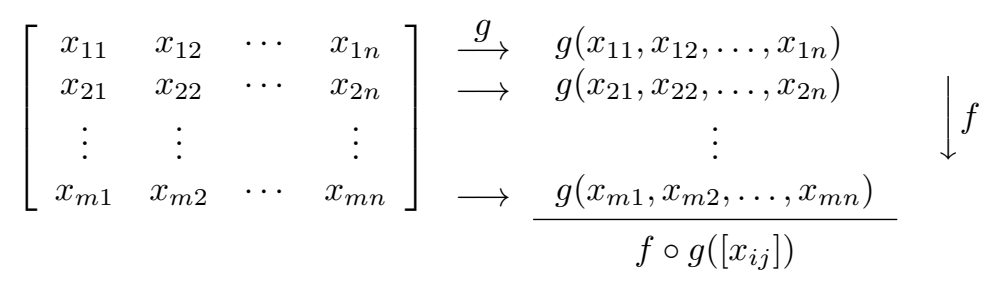

One says that $f$ commutes with $g$ on $\left[x_{i j}\right]$ if $f \circ g\left(\left[x_{i j}\right]\right)=g \circ f\left(\left[x_{i j}\right]^{\mathrm{T}}\right)$, and that $f$ and $g$ commute if they commute on all matrices $\left[x_{i j}\right] \in X^{m \times n}$. More explicitly, $f$ and $g$ commute on $X$ if

$$
\begin{aligned}
& f\left(g\left(x_{11}, x_{12}, \ldots, x_{1 n}\right), g\left(x_{21}, x_{22}, \ldots, x_{2 n}\right), \ldots, g\left(x_{m 1}, x_{m 2}, \ldots, x_{m n}\right)\right) \\
& \quad=g\left(f\left(x_{11}, x_{21}, \ldots, x_{m 1}\right), f\left(x_{12}, x_{22}, \ldots, x_{m 2}\right), \ldots, f\left(x_{1 n}, x_{2 n}, \ldots, x_{m n}\right)\right)
\end{aligned}
$$

holds for all $x_{i j} \in X(1 \leq i \leq m, 1 \leq j \leq n)$. A self-commuting operation is one that commutes with itself.

Operations that are self-commuting are also called entropic or medial. If $\mathcal{C}$ is a clone on $X$, then the set of operations on $X$ that commute with each member of $\mathcal{C}$ is another clone on $X$, called the centralizer of $\mathcal{C}$. Centralizer clones are also called bicentrally closed clones. On a finite set $X$, bicentrally closed clones coincide with primitive positive clones. There is a vast literature about entropic algebras, centralizer clones, and clones consisting of pairwise commuting operations. For entropic algebras, see [13] and the references therein. For commutative clones or centralizer clones see, for example, $[8,9,11,14]$. For primitive positive clones see $[2,7,10,15,16]$.

Date: November 1, 2010.

1991 Mathematics Subject Classification. Primary 08A40, 06D99; Secondary 39B05.

Key words and phrases. Polynomial operation, commuting operations, functional equation.

This material is based upon work supported by the Hungarian National Foundation for Scientific Research (OTKA) grant no. K77409. 
Aggregation functions return a single representative value from a list of values (such as the maximum or average of a list of real numbers). To aggregate the values in a table, one might use a row aggregation function and a (possibly different) column aggregation function. The commutativity of the row and column aggregation functions asserts that the final value is independent of the order of aggregation. A self-commuting aggregation function is called bisymmetric, and certain sequences of pairwise commuting aggregation functions are called strongly bisymmetric. See $[1,6]$ for more details.

It is easy to determine which pairs of module polynomial operations commute. Suppose that $M$ is an $R$-module, and that $f\left(x_{1}, \ldots, x_{m}\right)=\sum_{i=1}^{m} a_{i} x_{i}+c$ and $g\left(x_{1}, \ldots, x_{n}\right)=\sum_{j=1}^{n} b_{j} x_{j}+d$ are module polynomial operations. If $f$ and $g$ commute on the zero matrix, then it must be that (i) $\left(\sum_{i=1}^{m} a_{i}\right) d+c=\left(\sum_{j=1}^{n} b_{j}\right) c+d$ holds. If $f \circ g\left(\left[x_{i j}\right]\right)=g \circ f\left(\left[x_{i j}\right]^{\mathrm{T}}\right)$ on just those matrices $\left[x_{i j}\right]$ whose only nonzero entry is in position $i j$, then (ii) $\left(a_{i} b_{j}-b_{j} a_{i}\right) x=0$ holds for all $x \in M$. Conversely, if (i) and (ii) hold, then $f$ and $g$ commute on all matrices in $M^{m \times n}$.

The main features of the argument for module polynomials are: a normal form for polynomial operations is used and final results are expressed in terms of this normal form; a commutativity condition on coefficients of the normal form must hold and a condition on the constant term must hold; the commutativity of polynomials on general matrices is equivalent to commutativity on matrices with at most one nonzero entry. All of these features have analogues in our argument for commuting distributive lattice polynomial operations. Our result will be expressed in terms of the disjunctive normal form for polynomial operations. If $f\left(x_{1}, \ldots, x_{m}\right)=\sum_{S \subseteq[m]} a_{S} \prod_{i \in S} x_{i}$ and $g\left(x_{1}, \ldots, x_{n}\right)=\sum_{T \subseteq[n]} b_{T} \prod_{j \in T} x_{j}$ are distributive lattice polynomial operations written in disjunctive normal form, then $f$ commutes with $g$ if and only if (i) some condition on constant terms and leading coefficients is met and (ii) some type of "commutativity condition" is satisfied by all coefficients. Condition (i) is $a_{\emptyset}+b_{\emptyset} \leq a_{[m]} b_{[n]}$, which asserts that the join of the constant terms is dominated by the meet of the leading coefficients. This is equivalent to the condition that the ranges of $f$ and $g$ have nonempty intersection. This is obviously a necessary condition for $f$ to commute with $g$, and is equivalent to the commutativity of $f$ and $g$ on the zero matrix. The commutativity condition for the other coefficients in the case when $a_{\emptyset}=b_{\emptyset}=0$ is

$$
\begin{aligned}
a_{U_{1} \cap U_{2}} b_{V_{1} \cup V_{2}}+a_{U_{1}} b_{V_{1}} & +a_{U_{2}} b_{V_{2}}+a_{U_{1} \cup U_{2}} b_{V_{1}} b_{V_{2}} \\
& =a_{U_{1} \cup U_{2}} b_{V_{1} \cap V_{2}}+a_{U_{1}} b_{V_{1}}+a_{U_{2}} b_{V_{2}}+a_{U_{1}} a_{U_{2}} b_{V_{1} \cup V_{2}}
\end{aligned}
$$

for all $U_{1}, U_{2} \subseteq[m]$ and $V_{1}, V_{2} \subseteq[n]$. This condition can be shown to hold provided $f$ and $g$ commute on all 0,1-matrices where the 1's occur precisely in the union of two rectangular subregions $U_{1} \times V_{1}, U_{2} \times V_{2} \subseteq[m] \times[n]$. Conversely, we show that any pair of polynomial operations that commute on these "2-rectangle" matrices consisting solely of 0 's and 1's must commute on all matrices. Still under the assumption that $a_{\emptyset}=b_{\emptyset}=0$, we show that (1.2) is equivalent to the simpler condition

$$
a_{U_{1}} a_{U_{2}} b_{V}=a_{U_{1} \cap U_{2}} b_{V}+a_{U_{1}} a_{U_{2}} \sum_{v \in V} b_{\{v\}},
$$

together with the condition obtained from this by interchanging the roles of the $a$ 's and the $b$ 's. 
Corollaries of the main theorem include: a characterization of the self-commuting, distributive lattice polynomial operations (generalizing the results of [3]), and a characterization of the pairs of commuting distributive lattice term operations.

The main result of this paper was obtained after the BLAST 2010 conference held at the University of Colorado at Boulder. At this meeting, the report on [3] generated the question that is answered in this paper.

\section{Preliminaries}

Throughout the paper $[n]$ denotes $\{1,2, \ldots, n\}$ if $n$ is a natural number, $\mathcal{P}(X)$ denotes the power set of a set $X, \subseteq$ denotes inclusion, and $\subset$ denotes proper inclusion for sets.

The join and meet operations of a lattice will be denoted by + and · (or juxtaposition), respectively. If a lattice has a least element, then it will be denoted by 0 , and if it has a largest element, it will be denoted by 1 . If $L$ is a lattice, then $L^{01}$ denotes the smallest bounded lattice that contains $L$ as a sublattice; that is, $L^{01}=L \cup\{0,1\}$ where 0 is the least element of $L$ if $L$ has a least element, while 0 is a new least element otherwise, and similarly for 1 . It is straightforward to check that for a distributive lattice $L$ the lattice $L^{01}$ is also distributive.

Recall that a clone of operations on a set $X$ is a set of operations on $X$ that contains the projection operations and is closed under composition. The clone of polynomial operations of an algebra on $X$ is the least clone on $X$ that contains the fundamental operations of the algebra and all constant operations on $X$. If $L$ is a distributive lattice, then these conditions are satisfied by the collection of all operations on $L$ which can be written as a join of meets

$$
\sum_{S \in \mathcal{S}} M_{S} \quad \text { with } \quad M_{S}=\prod_{i \in S} x_{i} \text { or } M_{S}=\tilde{a}_{S} \prod_{i \in S} x_{i} \text { for each } S \in \mathcal{S},
$$

where $\mathcal{S}$ is a nonempty set of subsets of $[m]$ for some $m \geq 1, \tilde{a}_{S} \in L$ for all $S \in \mathcal{S}$, and $S \neq \emptyset$ if $M_{S}=\prod_{i \in S} x_{i}$. Allowing all elements of $L^{01}$ to be coefficients $\tilde{a}_{S}$, we can write every meet $M_{S}=\prod_{i \in S} x_{i}$ above as $M_{S}=\tilde{a}_{S} \prod_{i \in S} x_{i}$ with $\tilde{a}_{S}=1$, and we can expand the join $\sum_{S \in \mathcal{S}} M_{S}$ by joinands $M_{S}=\tilde{a}_{S} \prod_{i \in S} x_{i}$ with $\tilde{a}_{S}=0$ whenever $S \subseteq[m]$ but $S \notin \mathcal{S}$. Thus we get the following.

Lemma 2.1. If $L$ is a distributive lattice, then the clone of polynomial operations of $L$ consists of all operations of the form

$$
f\left(x_{1}, \ldots, x_{m}\right)=\sum_{S \subseteq[m]} \tilde{a}_{S} \prod_{i \in S} x_{i} \quad \text { with } m \geq 1 \text { and } \tilde{a}_{S} \in L^{01} \text { for all } S \subseteq[m]
$$

such that $\tilde{a}_{\emptyset} \neq 1$ if $1 \notin L$, and at least one coefficient $\tilde{a}_{S} \neq 0$ if $0 \notin L$.

For a distributive lattice $L$, we will denote the clone of polynomial operations of $L$ by $\operatorname{PClo}(L)$, and for each $f \in \operatorname{PClo}(L)$, we will refer to a representation of $f$ described in Lemma 2.1 as a disjunctive normal form, or briefly, a DNF of $f$. The joinand $\tilde{a}_{S} \prod_{i \in S} x_{i}$ of a DNF will be called the $S$-term, and $\tilde{a}_{S}$ the $S$-coefficient of the DNF. If $S=\{i\}$ is a singleton, then we will write $\tilde{a}_{i}$ instead of $\tilde{a}_{\{i\}}$.

An operation in $\mathrm{PClo}(L)$ can have many different DNFs. We will call a DNF

$$
f\left(x_{1}, \ldots, x_{m}\right)=\sum_{S \subseteq[m]} a_{S} \prod_{i \in S} x_{i}
$$


of $f$ maximal if the following conditions hold for the coefficients $a_{S}$ :

$$
a_{S} \leq a_{T} \quad \text { whenever } \quad S \subseteq T \subseteq[m],
$$

and

$$
a_{\emptyset} \neq 1 \text { if } 1 \notin L \text { and } \quad a_{[m]} \neq 0 \text { if } 0 \notin L .
$$

The next proposition shows that every polynomial operation $f$ of a distributive lattice has a unique maximal DNF. Moreover, it shows that for every $S \subseteq[\mathrm{m}]$, the $S$-coefficient of the maximal DNF of $f$ dominates the $S$-coefficients of all DNFs of $f$, which justifies the name "maximal DNF". For bounded distributive lattices, the construction of the maximal DNF of $f$ described in part (3) of the proposition can be found in [5].

Proposition 2.2. Let $L$ be a distributive lattice, and let $f$ be an $m$-ary polynomial operation of $L$ with DNF $f\left(x_{1}, \ldots, x_{m}\right)=\sum_{S \subseteq[m]} \tilde{a}_{S} \prod_{i \in S} x_{i}$.

(1) For the elements $a_{S}:=\sum_{Q \subseteq S} \tilde{a}_{Q}(S \subseteq[m])$ of $L^{01}$, (2.2) is a maximal DNF of $f$.

(2) The coefficients $a_{S}(S \subseteq[m])$ of a maximal DNF of $f$ are uniquely determined by $f$.

(3) If $L$ is bounded, then the coefficients of the maximal DNF of $f$ can be computed from $f$ as follows: for every $S \subseteq[m]$,

$$
a_{S}=f\left(e_{1}, \ldots, e_{m}\right) \quad \text { where } e_{i}=1 \text { if } i \in S \text { and } e_{i}=0 \text { if } i \notin S .
$$

Proof. We start the proof of (1) by verifying the equality (2.2). For all elements $x_{1}, \ldots, x_{n} \in L$, we have

$$
\sum_{S \subseteq[m]} a_{S} \prod_{i \in S} x_{i}=\sum_{S \subseteq[m]} \sum_{Q \subseteq S} \tilde{a}_{Q} \prod_{i \in S} x_{i}=\sum_{Q \subseteq[m]} \tilde{a}_{Q} \prod_{i \in Q} x_{i}=f\left(x_{1}, \ldots, x_{m}\right),
$$

where the first equality follows from the definition of $a_{S}$, while the second one follows from the absorption laws and the fact that $\tilde{a}_{Q} \prod_{i \in S} x_{i} \leq \tilde{a}_{Q} \prod_{i \in Q} x_{i}$ whenever $Q \subseteq S$. This proves (2.2). Condition (2.3) follows immediately from the definition of $a_{S}$. Finally, since $a_{\emptyset}=\tilde{a}_{\emptyset}$ and $a_{[m]}=\sum_{Q \subseteq[m]} \tilde{a}_{Q}$, condition (2.4) is just a restatement of the restrictions on $\tilde{a}_{S}$ stated in Lemma 2.1. Thus, the proof of (1) is complete.

For $(2)$, let $f\left(x_{1}, \ldots, x_{m}\right)=\sum_{S \subseteq[m]} a_{S}^{\prime} \prod_{i \in S} x_{i}$ be another maximal DNF of $f$, and assume that $a_{U} \neq a_{U}^{\prime}$ for some $U \subseteq[m]$. By symmetry, we may assume that $a_{U}^{\prime} \not \leq a_{U}$. Thus $a_{U} \neq 1$ and $a_{U}^{\prime} \neq 0$. Now choose elements $c, d \in L$ such that

$$
c \geq a_{U}, \quad d \leq a_{U}^{\prime}, \quad \text { and } \quad d \not \leq c .
$$

If $a_{U}$ and $a_{U}^{\prime}$ are in $L$, then we can let $c=a_{U}$ and $d=a_{U}^{\prime}$, but $c, d \in L$ satisfying (2.5) exist even if $a_{U}=0 \notin L$ or $a_{U}^{\prime}=1 \notin L$. This is so because $a_{U}=0 \notin L$ and $a_{U}^{\prime} \neq 0$ imply that the principal ideal ( $\left.d\right]$ of $L$ is infinite for all $d \in L, d \leq a_{U}^{\prime}$. Thus there exist $c, d \in L$ such that $0=a_{U} \leq c<d \leq a_{U}^{\prime}$. A dual argument works if $a_{U}^{\prime}=1 \notin L$. For $1 \leq i \leq m$ let $d_{i}=d$ if $i \in U$ and $d_{i}=c$ if $i \notin U$. Then, from the first maximal DNF of $f$ we get that

$$
f\left(d_{1}, \ldots, d_{m}\right)=a_{\emptyset}+\sum_{\emptyset \neq S \subseteq U} a_{S} d+\sum_{S \nsubseteq U} a_{S} c \leq a_{\emptyset}+a_{U} d+a_{[m]} c \leq a_{U}+c=c,
$$


where the inequalities $\leq$ follow from the monotonicity (2.3) of the coefficients $a_{S}$, and the last equality follows from $c \geq a_{U}$ in (2.5). From the second maximal DNF of $f$ we obtain that

$$
f\left(d_{1}, \ldots, d_{m}\right)=a_{\emptyset}^{\prime}+\sum_{\emptyset \neq S \subset U} a_{S}^{\prime} d+a_{U}^{\prime} d+\sum_{S \nsubseteq U} a_{S}^{\prime} c \geq a_{U}^{\prime} d=d,
$$

where the last equality follows from $d \leq a_{U}^{\prime}$ in (2.5). The last two displayed inequalitites yield that $d \leq c$. However, $c$ and $d$ were chosen in (2.5) so that $d \not \leq c$. Thus we reached the desired contradiction, which completes the proof of (2).

Finally, if $L$ is bounded and $S \subseteq[m]$, then for $e_{1}, \ldots, e_{m}$ as in (3),

$$
f\left(e_{1}, \ldots, e_{m}\right)=a_{\emptyset}+\sum_{\emptyset \neq S^{\prime} \subseteq S} a_{S^{\prime}} \cdot 1+\sum_{S^{\prime} \nsubseteq S} a_{S^{\prime}} \cdot 0=\sum_{S^{\prime} \subseteq S} a_{S^{\prime}}=a_{S},
$$

by $(2.3)$.

Proposition 2.2 implies that if in a DNF $f\left(x_{1}, \ldots, x_{m}\right)=\sum_{S \subset[m]} \tilde{a}_{S} \prod_{i \in S} x_{i}$ of $f \in \operatorname{PClo}(L)$ we have $\tilde{a}_{U} \leq \sum_{Q \subset U} \tilde{a}_{Q}$ for some $U \subseteq[m]$, then by omitting the joinand $\tilde{a}_{U} \prod_{i \in U} x_{i}$ (i.e., replacing $\tilde{a}_{U}$ by 0 ) we still have a DNF for $f$, because the two DNFs yield the same maximal DNF. This justifies the following definition.

If $L$ is a distributive lattice and $f$ is a polynomial operation of $L$ then the $U$-term $\tilde{a}_{U} \prod_{i \in U} x_{i}$ of a DNF $f\left(x_{1}, \ldots, x_{m}\right)=\sum_{S \subseteq[m]} \tilde{a}_{S} \prod_{i \in S} x_{i}$ of $f$ will be called inessential if $\tilde{a}_{U} \leq \sum_{Q \subset U} \tilde{a}_{Q}$, and essential otherwise. In the maximal DNF $f\left(x_{1}, \ldots, x_{m}\right)=\sum_{S \subseteq[m]} a_{S} \prod_{i \in S} x_{i}$ of $f$ we have $a_{U} \geq \sum_{Q \subset U} a_{Q}$ for every $U \subseteq[m]$, therefore the $U$-term is essential if and only if $a_{U}>\sum_{Q \subset U} a_{Q}$.

For a distributive lattice $L$ let

$\operatorname{PClo}^{*}\left(L^{01}\right):=$

$\left\{f \in \operatorname{PClo}\left(L^{01}\right): f\right.$ is not a constant operation with value in $\left.L^{01} \backslash L\right\}$.

The existence and uniqueness of maximal DNFs for polynomial operations of $L$ immediately implies the following corollary.

Corollary 2.3. For every distributive lattice $L$, the map $\mathrm{PClo}(L) \rightarrow \mathrm{PClo}^{*}\left(L^{01}\right)$ that assigns to every polynomial operation $f \in \operatorname{PClo}(L)$ the polynomial operation $f^{*} \in \mathrm{PClo}^{*}\left(L^{01}\right)$ which has the same maximal DNF as $f$, is a clone isomorphism. Consequently, every polynomial operation $f$ of $L$ has a unique extension to a polynomial operation of $L^{01}$, namely $f^{*}$.

\section{Commuting polynomial operations of Distributive lattices}

Recall from the introduction that two operations $f$ and $g$ on a set $X$ commute if they satisfy the equality (1.1) for all arguments $x_{i j} \in X$. We will write $f \perp g$ to indicate that $f$ and $g$ commute. Clearly, $f \perp g$ if and only if $g \perp f$.

From now on $f, g$ will be polynomial operations of a distributive lattice. First we will rewrite the condition defining $f \perp g$ in terms of the maximal DNFs of $f$ and $g$. The following notation will be useful: if $R \subseteq[m] \times[n](m, n \geq 1)$, then for arbitrary $i \in[m]$ and $j \in[n]$ we let

$$
\begin{aligned}
& R(i,-):=\{j \in[n]:(i, j) \in R\} \quad \text { and } \\
& R(-, j):=\{i \in[m]:(i, j) \in R\} .
\end{aligned}
$$


Proposition 3.1. If $L$ is a distributive lattice and $f, g$ are polynomial operations of $L$ with maximal DNFs

$$
f\left(x_{1}, \ldots, x_{m}\right)=\sum_{S \subseteq[m]} a_{S} \prod_{i \in S} x_{i} \quad \text { and } \quad g\left(x_{1}, \ldots, x_{n}\right)=\sum_{T \subseteq[n]} b_{T} \prod_{j \in T} x_{j},
$$

then $f \perp g$ if and only if the following equality holds for all $R \subseteq[m] \times[n]$ :

$$
\sum_{S \subseteq[m]} a_{S} \prod_{i \in S} b_{R(i,-)}=\sum_{T \subseteq[n]} b_{T} \prod_{j \in T} a_{R(-, j)} .
$$

Proof. By definition, $f$ and $g$ commute if and only if the $m n$-ary composite polynomials $f \circ g\left(\left[x_{i j}\right]\right)$ and $g \circ f\left(\left[x_{i j}\right]^{\mathrm{T}}\right)$ shown on the two sides of (1.1) are equal. Our goal is to prove that for each $R \subseteq[m] \times[n]$, the left-hand side of (3.1) is the $R$-coefficient of the maximal DNF of $f \circ g\left(\left[x_{i j}\right]\right)$, while the right-hand side of (3.1) is the $R$-coefficient of the maximal DNF of $g \circ f\left(\left[x_{i j}\right]^{\mathrm{T}}\right)$. This will imply that the equality (3.1) holds for all $R \subseteq[m] \times[n]$ if and only if $f \circ g\left(\left[x_{i j}\right]\right)$ and $g \circ f\left(\left[x_{i j}\right]^{\mathrm{T}}\right)$ have the same maximal DNFs, i.e., $f \circ g\left(\left[x_{i j}\right]\right)$ and $g \circ f\left(\left[x_{i j}\right]^{\mathrm{T}}\right)$ are the same polynomial operation, and will therefore complete the proof.

Using the maximal DNFs of $f$ and $g$ we see that

$$
\begin{aligned}
f \circ g\left(\left[x_{i j}\right]\right) & =\sum_{S \subseteq[m]} a_{S} \prod_{i \in S}\left(\sum_{T \subseteq[n]} b_{T} \prod_{j \in T} x_{i j}\right) \\
& =\sum_{S \subseteq[m]} \sum_{\left(T_{i}\right)_{i \in S} \in \mathcal{P}([n])^{S}} a_{S} \prod_{i \in S} b_{T_{i}}\left(\prod_{i \in S} \prod_{j \in T_{i}} x_{i j}\right) .
\end{aligned}
$$

This yields a DNF for $f \circ g\left(\left[x_{i j}\right]\right)$ in which, for each $R \subseteq[m] \times[n]$, the $R$-coefficient is

$$
\tilde{c}_{R}:=\sum_{S \subseteq[m]} a_{S} \prod_{i \in S} b_{R(i,-)} .
$$

If $R^{\prime} \subseteq R$, then for each $i \in[m]$ we have that $R^{\prime}(i,-) \subseteq R(i,-)$, and hence the inequality $b_{R^{\prime}(i,-)} \leq b_{R(i,-)}$ holds. This implies that $\tilde{c}_{R^{\prime}} \leq \tilde{c}_{R}$. Therefore, it follows from Proposition 2.2 (1) that the $R$-coefficient of the maximal DNF of $f \circ g\left(\left[x_{i j}\right]\right)$ is $\sum_{R^{\prime} \subseteq R} \tilde{c}_{R}^{\prime}=\tilde{c}_{R}$, which is the left-hand side of (3.1).

The fact that the $R$-coefficient of the maximal DNF of $g \circ f\left(\left[x_{i j}\right]^{\mathrm{T}}\right)$ is the righthand side of (3.1) follows from the result in the preceding paragraph by observing that the operation $g \circ f\left(\left[x_{i j}\right]^{\mathrm{T}}\right)$ is obtained from $f \circ g\left(\left[x_{i j}\right]\right)$ by switching the roles of $f$ and $g$ and simultaneously switching the subscripts of the variables.

Corollary 3.2. Let $L$ be a distributive lattice, let $f, g \in \operatorname{PClo}(L)$, and let $C$ be the sublattice of $L^{01}$ generated by the coefficients of the maximal DNFs of $f$ and $g$. The following conditions on $f$ and $g$ are equivalent:

(i) $f$ and $g$ are commuting polynomial operations of $L$;

(ii) the unique extensions $f^{*}$ and $g^{*}$ of $f$ and $g$ to $L^{01}$ are commuting polynomial operations of $L^{01}$

(iii) the restrictions $\left.f^{*}\right|_{C}$ and $\left.g^{*}\right|_{C}$ of $f^{*}$ and $g^{*}$ to $C$ are commuting polynomial operations of the finite lattice $C$.

Proof. Clearly, the lattice $C$ is finitely generated, hence it is finite. We will use the notation of Proposition 3.1 for the maximal DNFs of $f$ and $g$. By the definition of $f^{*}, f^{*}$ has the same maximal DNF as $f$. Furthermore, since $C$ contains the coefficients of the maximal DNF of $f^{*}, f^{*}$ can be restricted to $C$, and $\left.f^{*}\right|_{C}$ is a 
polynomial operation of $C$ with the same maximal DNF as $f^{*}$ (and $f$ ). Similarly, $g^{*} \in \operatorname{PClo}\left(L^{01}\right)$ and $\left.g^{*}\right|_{C} \in \operatorname{PClo}(C)$ have the same maximal DNF as $g$. Therefore, by Proposition 3.1, each one of the commutativity conditions $f \perp g, f^{*} \perp g^{*}$, and $\left.\left.f^{*}\right|_{C} \perp g^{*}\right|_{C}$ is equivalent to the requirement that (3.1) holds for the coefficients of their maximal DNFs for all $R \subseteq[m] \times[n]$. It follows that (i) $\Leftrightarrow$ (ii) $\Leftrightarrow$ (iii).

Corollary 3.2 shows that when studying the relation $f \perp g$ for polynomial operations $f, g$ of distributive lattices one could restrict, without loss of generality, to bounded distributive lattices, or even to finite lattices.

Next we will establish some necessary conditions for two polynomial operations to commute. The equivalence of some of conditions (i)-(vi) below for unary polynomial operations of distributive lattices appears in [4].

Lemma 3.3. Let $f, g$ be polynomial operations of a distributive lattice $L$ with maximal DNFs as in Proposition 3.1. If $f$ and $g$ commute, then they must satisfy the following equivalent conditions:

(i) the unary polynomial operations $f(x, \ldots, x)$ and $g(x, \ldots, x)$ commute;

(ii) $a_{\emptyset}+a_{[m]} b_{\emptyset}=b_{\emptyset}+b_{[n]} a_{\emptyset}$;

(iii) the coefficients of the maximal DNFs of $f$ and $g$ satisfy (3.1) for $R=\emptyset$;

(iv) $\operatorname{im}(f) \cap \operatorname{im}(g) \neq \emptyset$;

(v) $a_{\emptyset}+b_{\emptyset} \leq a_{[m]} b_{[n]}$;

(vi) $a_{\emptyset}+a_{[m]} b_{\emptyset}=a_{\emptyset}+b_{\emptyset}=b_{\emptyset}+b_{[n]} a_{\emptyset}$.

Proof. If $f$ and $g$ commute on all matrices, then they commute on all constant matrices, which is easily seen to be equivalent to condition (i). It remains to show that all conditions are equivalent. First we make some remarks about distributive lattice polynomials.

Since $a_{\emptyset}$ is a joinand of the maximal DNF for $f$, it follows that $a_{\emptyset} \leq c$ holds for all $c \in \operatorname{im}(f)$. Since $f$ and $a_{[m]} f$ have the same maximal DNF, it follows that $c \leq a_{[m]}$ for all $c \in \operatorname{im}(f)$. Hence $\operatorname{im}(f)$ is contained in the restriction to $L$ of the interval $\left[a_{\emptyset}, a_{[m]}\right]$ of $L^{01}$. On the other hand, for any $d \in\left[a_{\emptyset}, a_{[m]}\right] \cap L$ it is the case that $f(d, \ldots, d)=d$, so the set $\operatorname{im}(f)$ exactly coincides with $\left[a_{\emptyset}, a_{[m]}\right] \cap L$.

Now we begin the proof. Assume (i), so that $f^{\prime}(x):=f(x, \ldots, x)=a_{\emptyset}+a_{[m]} x$ and $g^{\prime}(x)=b_{\emptyset}+b_{[n]} x$ commute. The equality between $f^{\prime} \circ g^{\prime}([x])$ and $g^{\prime} \circ f^{\prime}([x])$ is expressible as

$$
\left(a_{\emptyset}+a_{[m]} b_{\emptyset}\right)+a_{[m]} b_{[n]} x=\left(b_{\emptyset}+b_{[n]} a_{\emptyset}\right)+a_{[m]} b_{[n]} x,
$$

which holds if and only if $a_{\emptyset}+a_{[m]} b_{\emptyset}=b_{\emptyset}+b_{[n]} a_{\emptyset}$. Hence (i) $\Leftrightarrow$ (ii).

If $R=\emptyset$ in (3.1), then, due to the monotonicity of the coefficients, (3.1) reduces to $a_{\emptyset}+a_{[m]} b_{\emptyset}=b_{\emptyset}+b_{[n]} a_{\emptyset}$. Hence (ii) $\Leftrightarrow$ (iii).

Condition (iii) asserts that $f \circ g\left(\left[x_{i j}\right]\right)$ and $g \circ f\left(\left[x_{i j}\right]^{\mathrm{T}}\right)$ have the same constant term $c$. Let $I_{f g}$ and $I_{g f}$ denote the images of $f \circ g\left(\left[x_{i j}\right]\right)$ and $g \circ f\left(\left[x_{i j}\right]^{\mathrm{T}}\right)$. If $c \in L$, then by the observations of the second paragraph of this proof we have that $c \in I_{f g} \subseteq \operatorname{im}(f)$ and $c \in I_{g f} \subseteq \operatorname{im}(g)$. It follows that $c \in \operatorname{im}(f) \cap \operatorname{im}(g)$. If, on the other hand, $c=0 \notin L$, then both $I_{f g}$ and $I_{g f}$ are nonempty downward directed sets. Hence if $d \in I_{f g}$ and $e \in I_{g f}$, then $d e \in I_{f g} \cap I_{g f} \subseteq \operatorname{im}(f) \cap \operatorname{im}(g)$. This shows that (iii) implies (iv).

Assume that (iv) holds. If $d \in \operatorname{im}(f) \cap \operatorname{im}(g)=\left[a_{\emptyset}, a_{[m]}\right] \cap\left[b_{\emptyset}, b_{[n]}\right] \cap L$, then $a_{\emptyset}+b_{\emptyset} \leq d \leq a_{[m]} b_{[n]}$. Hence (iv) implies (v). 
If (v) holds, then

$$
\begin{aligned}
a_{\emptyset}+a_{[m]} b_{\emptyset} & =\left(a_{\emptyset}+a_{[m]}\right)\left(a_{\emptyset}+b_{\emptyset}\right) \\
& =a_{[m]}\left(a_{\emptyset}+b_{\emptyset}\right) \\
& =a_{\emptyset}+b_{\emptyset},
\end{aligned}
$$

where the first equality follows from distributivity, the second follows from the monotonicity of coefficients in a maximal DNF, and the third follows from (v): $a_{\emptyset}+b_{\emptyset} \leq a_{[m]} b_{[n]}\left(\leq a_{[m]}\right)$. By symmetry we also have $b_{\emptyset}+b_{[n]} a_{\emptyset}=a_{\emptyset}+b_{\emptyset}$, so (v) implies (vi).

Item (ii) is included in (vi), so we are done.

Lemma 3.4. Let $f, g$ be polynomial operations of a distributive lattice $L$ with maximal DNFs as in Proposition 3.1. For arbitrary subsets $U_{1}, U_{2} \subseteq[m]$ and $V_{1}, V_{2} \subseteq[n]$, the equality (3.1) for $R=\left(U_{1} \times V_{1}\right) \cup\left(U_{2} \times V_{2}\right)$ is equivalent to the equality

$$
\begin{gathered}
a_{\emptyset}+a_{[m]} b_{\emptyset}+a_{U_{1} \cap U_{2}} b_{V_{1} \cup V_{2}}+a_{U_{1}} b_{V_{1}}+a_{U_{2}} b_{V_{2}}+a_{U_{1} \cup U_{2}} b_{V_{1}} b_{V_{2}}= \\
b_{\emptyset}+b_{[n]} a_{\emptyset}+b_{V_{1} \cap V_{2}} a_{U_{1} \cup U_{2}}+b_{V_{1}} a_{U_{1}}+b_{V_{2}} a_{U_{2}}+b_{V_{1} \cup V_{2}} a_{U_{1}} a_{U_{2}} .
\end{gathered}
$$

Consequently, (3.2) holds for $f$ and $g$ whenever $f$ and $g$ commute.

Since (3.2) is obtained from the special case of (3.1) when $R$ is a union of two rectangles $U_{i} \times V_{i}(i=1,2)$, we will refer to (3.2) as the 2-rectangle condition.

Proof. Throughout the proof, $U_{1}, U_{2} \subseteq[m]$ and $V_{1}, V_{2} \subseteq[n]$ are fixed, and $R=$ $\left(U_{1} \times V_{1}\right) \cup\left(U_{2} \times V_{2}\right)$. First we will simplify the left-hand side of (3.1) for this $R$. We want to show that

$$
\begin{aligned}
\sum_{S \subseteq[m]} a_{S} & \prod_{i \in S} b_{R(i,-)} \\
& =a_{\emptyset}+a_{[m]} b_{\emptyset}+a_{U_{1} \cap U_{2}} b_{V_{1} \cup V_{2}}+a_{U_{1}} b_{V_{1}}+a_{U_{2}} b_{V_{2}}+a_{U_{1} \cup U_{2}} b_{V_{1}} b_{V_{2}} .
\end{aligned}
$$

We will use the monotonicity of the coefficients of the maximal DNFs of $f$ and $g$, namely that (2.3) holds for the $a$ 's, and analogously, for the $b$ 's. Also, notice that the shape of $R$ implies that

$$
R(i,-)= \begin{cases}V_{1} \cup V_{2} & \text { if } i \in U_{1} \cap U_{2}, \\ V_{\ell} & \text { if } i \in U_{\ell} \backslash\left(U_{1} \cap U_{2}\right), \ell=1,2, \text { and } \\ \emptyset & \text { if } i \in[m] \backslash\left(U_{1} \cup U_{2}\right) .\end{cases}
$$

The fact that the left-hand side of (3.3) is dominated by the right-hand side will follow if we verify that every joinand on the left-hand side is $\leq$ a joinand on the right-hand side. Let $S \subseteq[m]$. If $S=\emptyset$, then $a_{S} \prod_{i \in S} b_{R(i,-)}=a_{\emptyset}$, which is a joinand on the right-hand side. If $S \neq \emptyset$ but $S \subseteq U_{1} \cap U_{2}$, then by the description of $R(i,-)$ above we have that $R(i,-)=V_{1} \cup V_{2}$ for each $i \in S$. Since $S \neq \emptyset$, the monotonicity of the $a$ 's and $b$ 's implies that

$$
a_{S} \prod_{i \in S} b_{R(i,-)} \leq a_{U_{1} \cap U_{2}} \prod_{i \in S} b_{V_{1} \cup V_{2}}=a_{U_{1} \cap U_{2}} b_{V_{1} \cup V_{2}} .
$$


If $S \nsubseteq U_{1} \cap U_{2}$ but $S \subseteq U_{\ell}$ for $\ell=1$ or 2 , then $R(i,-)=V_{1} \cup V_{2}$ for each $i \in S \cap\left(U_{1} \cap U_{2}\right)$, while $R(\bar{i},-)=V_{\ell}$ for each $i \in S \backslash\left(U_{1} \cap U_{2}\right)$. Since $S \backslash\left(U_{1} \cap U_{2}\right) \neq \emptyset$, the monotonicity of the $a$ 's and $b$ 's implies again that

$$
a_{S} \prod_{i \in S} b_{R(i,-)} \leq a_{U_{\ell}} \prod_{i \in S \cap\left(U_{1} \cap U_{2}\right)} b_{V_{1} \cup V_{2}} \prod_{i \in S \backslash\left(U_{1} \cap U_{2}\right)} b_{V_{\ell}}=a_{U_{\ell}} b_{V_{\ell}} .
$$

If $S \nsubseteq U_{1}, U_{2}$ but $S \subseteq U_{1} \cup U_{2}$, then $R(i,-)=V_{1} \cup V_{2}$ for each $i \in S \cap\left(U_{1} \cap U_{2}\right)$ and $R(i,-)=V_{\ell}$ for each $i \in\left(S \cap U_{\ell}\right) \backslash\left(S \cap U_{3-\ell}\right)(\ell=1,2)$. Since $\left(S \cap U_{\ell}\right) \backslash\left(S \cap U_{3-\ell}\right) \neq \emptyset$ for both $\ell=1,2$, the monotonicity of the $a$ 's and $b$ 's implies that

$$
\begin{aligned}
a_{S} \prod_{i \in S} b_{R(i,-)} & \leq a_{U_{1} \cup U_{2}} \prod_{i \in S \cap\left(U_{1} \cup U_{2}\right)} b_{V_{1} \cup V_{2}} \prod_{i \in\left(S \cap U_{1}\right) \backslash\left(S \cup U_{2}\right)} b_{V_{1}} \prod_{i \in\left(S \cap U_{2}\right) \backslash\left(S \cup U_{1}\right)} b_{V_{2}} \\
& =a_{U_{1} \cup U_{2}} b_{V_{1}} b_{V_{2}} .
\end{aligned}
$$

Finally, if $S \nsubseteq U_{1} \cup U_{2}$, then $R(i,-)=\emptyset$ for all $i \in S \backslash\left(U_{1} \cup U_{2}\right)$. Since $S \backslash\left(U_{1} \cup U_{2}\right) \neq \emptyset$, we get that

$$
a_{S} \prod_{i \in S} b_{R(i,-)} \leq a_{[m]} \prod_{i \in S \cap\left(U_{1} \cup U_{2}\right)} b_{R(i,-)} \prod_{i \in S \backslash\left(U_{1} \cup U_{2}\right)} b_{\emptyset}=a_{[m]} b_{\emptyset} .
$$

This proves $\leq$ in $(3.3)$

To prove the reverse inequality $\geq$ in (3.3) it suffices to establish that every joinand on the right-hand side is bounded above by a joinand $a_{S} \prod_{i \in S} b_{R(i,-)}$ on the left-hand side. The first joinand $a_{\emptyset}$ appears as $a_{S} \prod_{i \in S} b_{R(i,-)}$ for $S=\emptyset$. The last joinand satisfies $a_{U_{1} \cup U_{2}} b_{V_{1}} b_{V_{2}} \leq a_{U_{1} \cup U_{2}} \prod_{i \in U_{1} \cup U_{2}} b_{R(i,-)}$, because we have $R(i,-) \in\left\{V_{1}, V_{2}\right\}$ for each $i \in U_{1} \cup U_{2}$. All other joinands on the right-hand side of (3.3) are of the form $a_{S} b_{T}$ such that $S \in\left\{[m], U_{1} \cap U_{2}, U_{1}, U_{2}\right\}$ and $T \subseteq R(i,-)$ for all $i \in S$. Therefore, they satisfy $a_{S} b_{T} \leq a_{S} \prod_{i \in S} b_{R(i,-)}$.

This proves the equality (3.3), which simplifies the left-hand side of (3.1) for the set $R=\left(U_{1} \times V_{1}\right) \cup\left(U_{2} \times V_{2}\right)$. By switching the roles of $f$ and $g$ we get an analogous equality for the right-hand side of (3.1):

$$
\begin{aligned}
\sum_{T \subseteq[n]} b_{T} & \prod_{j \in T} a_{R(-, j)} \\
& =b_{\emptyset}+b_{[n]} a_{\emptyset}+b_{V_{1} \cap V_{2}} a_{U_{1} \cup U_{2}}+b_{V_{1}} a_{U_{1}}+b_{V_{2}} a_{U_{2}}+b_{V_{1} \cup V_{2}} a_{U_{1}} a_{U_{2}} .
\end{aligned}
$$

Thus we obtain from (3.3) and (3.4) that for $R=\left(U_{1} \times V_{1}\right) \cup\left(U_{2} \times V_{2}\right)$, condition (3.1) is equivalent to (3.2), as claimed.

After these preparations we can state the main theorem of this paper, which characterizes commuting pairs of polynomial operations of distributive lattices. We will show that two polynomial operations commute if and only if they satisfy the 2-rectangle condition (3.2). We will also present a more transparent condition characterizing commutativity. As the proof develops we will find other necessary and sufficient conditions for commutativity, which we will summarize in Corollary 3.10.

Theorem 3.5. Let $L$ be a distributive lattice, and let $f, g$ be polynomial operations of $L$ with maximal DNFs

$$
f\left(x_{1}, \ldots, x_{m}\right)=\sum_{S \subseteq[m]} a_{S} \prod_{i \in S} x_{i} \quad \text { and } \quad g\left(x_{1}, \ldots, x_{n}\right)=\sum_{T \subseteq[n]} b_{T} \prod_{j \in T} x_{j} .
$$

The following conditions on $f$ and $g$ are equivalent: 
(i) $f \perp g$;

(ii) the 2-rectangle condition (3.2) holds for all $U_{1}, U_{2} \subseteq[m]$ and $V_{1}, V_{2} \subseteq[n]$;

(iii) (a) $a_{\emptyset}+b_{\emptyset} \leq a_{[m]} b_{[n]}$ (i.e., $\operatorname{im}(f) \cap \operatorname{im}(g) \neq \emptyset$ ), and

(b) the equalities

$$
\begin{gathered}
a_{\emptyset}+a_{U_{1}} a_{U_{2}} b_{V}=a_{\emptyset}+a_{U_{1} \cap U_{2}} b_{V}+a_{U_{1}} a_{U_{2}}\left(b_{\emptyset}+\sum_{v \in V} b_{v}\right), \\
b_{\emptyset}+b_{V_{1}} b_{V_{2}} a_{U}=b_{\emptyset}+b_{V_{1} \cap V_{2}} a_{U}+b_{V_{1}} b_{V_{2}}\left(a_{\emptyset}+\sum_{u \in U} a_{u}\right) .
\end{gathered}
$$

hold for all $U_{1}, U_{2}, U \subseteq[m]$ and $V_{1}, V_{2}, V \subseteq[n]$.

The proof of Theorem 3.5 will occupy most of this section. Since the implication (i) $\Rightarrow$ (ii) has been established already in Lemma 3.4, we will first focus on the implication (ii) $\Rightarrow$ (iii)

Lemma 3.6. Let $f, g \in \mathrm{PClo}(L)$ be as in Theorem 3.5. If $f$ and $g$ satisfy condition (ii) in Theorem 3.5, then they also satisfy the following condition:

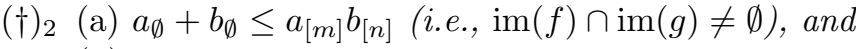

(b) the equalities

$$
\begin{aligned}
& a_{\emptyset}+a_{U_{1}} a_{U_{2}} b_{V_{1} \cup V_{2}}=a_{\emptyset}+a_{U_{1} \cap U_{2}} b_{V_{1} \cup V_{2}}+a_{U_{1}} a_{U_{2}}\left(b_{V_{1}}+b_{V_{2}}\right), \\
& b_{\emptyset}+b_{V_{1}} b_{V_{2}} a_{U_{1} \cup U_{2}}=b_{\emptyset}+b_{V_{1} \cap V_{2}} a_{U_{1} \cup U_{2}}+b_{V_{1}} b_{V_{2}}\left(a_{U_{1}}+a_{U_{2}}\right) \\
& \text { hold for all } U_{1}, U_{2} \subseteq[m] \text { and } V_{1}, V_{2} \subseteq[n] .
\end{aligned}
$$

Proof. Assume that the 2-rectangle condition (3.2) holds for all $U_{1}, U_{2} \subseteq[m]$ and $V_{1}, V_{2} \subseteq[n]$. By Lemma 3.4, condition (3.2) for $U_{1}=U_{2}=V_{1}=V_{2}=\emptyset$ is equivalent to condition (3.1) for $R=\emptyset$, and by Lemma 3.3, the latter is equivalent to the inequality $a_{\emptyset}+b_{\emptyset} \leq a_{[m]} b_{[n]}$, as well as to the condition that $\operatorname{im}(f) \cap \operatorname{im}(g) \neq \emptyset$. This proves (a).

For (b), to prove (3.7) for arbitrary $U_{1}, U_{2} \subseteq[m]$ and $V_{1}, V_{2} \subseteq[n]$, we take the meet of the left-hand side of (3.2) with $a_{U_{1}} a_{U_{2}}$, and apply the distributive and absorption laws to get that

$$
\begin{aligned}
a_{U_{1}} a_{U_{2}}\left(a_{\emptyset}+a_{[m]} b_{\emptyset}+a_{U_{1} \cap U_{2}} b_{V_{1} \cup V_{2}}\right. & \left.+a_{U_{1}} b_{V_{1}}+a_{U_{2}} b_{V_{2}}+a_{U_{1} \cup U_{2}} b_{V_{1}} b_{V_{2}}\right) \\
=a_{\emptyset}+a_{U_{1}} a_{U_{2}} b_{\emptyset}+a_{U_{1} \cap U_{2}} b_{V_{1} \cup V_{2}} & +a_{U_{1}} a_{U_{2}} b_{V_{1}}+a_{U_{1}} a_{U_{2}} b_{V_{2}}+a_{U_{1}} a_{U_{2}} b_{V_{1}} b_{V_{2}} \\
& =a_{\emptyset}+a_{U_{1} \cap U_{2}} b_{V_{1} \cup V_{2}}+a_{U_{1}} a_{U_{2}}\left(b_{V_{1}}+b_{V_{2}}\right) .
\end{aligned}
$$

Taking the meet of the right-hand side of (3.2) with $a_{U_{1}} a_{U_{2}}$ and applying the distributive and absorption laws again we obtain that

$$
\begin{array}{r}
a_{U_{1}} a_{U_{2}}\left(b_{\emptyset}+b_{[n]} a_{\emptyset}+b_{V_{1} \cap V_{2}} a_{U_{1} \cup U_{2}}+b_{V_{1}} a_{U_{1}}+b_{V_{2}} a_{U_{2}}+b_{V_{1} \cup V_{2}} a_{U_{1}} a_{U_{2}}\right) \\
=b_{\emptyset} a_{U_{1}} a_{U_{2}}+b_{[n]} a_{\emptyset}+b_{V_{1} \cap V_{2}} a_{U_{1}} a_{U_{2}}+b_{V_{1}} a_{U_{1}} a_{U_{2}}+b_{V_{2}} a_{U_{1}} a_{U_{2}}+b_{V_{1} \cup V_{2}} a_{U_{1}} a_{U_{2}} \\
=b_{[n]} a_{\emptyset}+b_{V_{1} \cup V_{2}} a_{U_{1}} a_{U_{2}} .
\end{array}
$$

Thus (3.2) implies that

$$
b_{[n]} a_{\emptyset}+b_{V_{1} \cup V_{2}} a_{U_{1}} a_{U_{2}}=a_{\emptyset}+a_{U_{1} \cap U_{2}} b_{V_{1} \cup V_{2}}+a_{U_{1}} a_{U_{2}}\left(b_{V_{1}}+b_{V_{2}}\right) .
$$


Since $a_{\emptyset} \leq b_{[n]}$, and hence $b_{[n]} a_{\emptyset}=a_{\emptyset}$, the equality (3.7) follows. The equality (3.8) can be proved in a similar way.

Lemma 3.7. Let $f, g \in \operatorname{PClo}(L)$ be as in Theorem 3.5. If $f$ and $g$ satisfy condition $(\dagger)_{2}$ in Lemma 3.6, then they also satisfy condition (iii) in Theorem 3.5.

Proof. Assume that condition $(\dagger)_{2}$ in Lemma 3.6 holds for $f$ and $g$. We have to verify the equalities (3.5) and (3.6). Since (3.5) and (3.6) can be obtained from one another by interchanging the roles of the $a_{S}$ 's and the $b_{T}$ 's (i.e., the roles of $f$ and $g$ ), it is enough to prove (3.5). Let $U_{1}, U_{2} \subseteq[m]$ be fixed, and let $V \subseteq[n]$. We will prove the equality (3.5) by induction on $|V|$. For $V=\emptyset(3.5)$ is the equality

$$
a_{\emptyset}+a_{U_{1}} a_{U_{2}} b_{\emptyset}=a_{\emptyset}+a_{U_{1} \cap U_{2}} b_{\emptyset}+a_{U_{1}} a_{U_{2}} b_{\emptyset},
$$

which is clearly true, as $a_{U_{1} \cap U_{2}} \leq a_{U_{1}} a_{U_{2}}$.

Next let $|V| \geq 1$, say $V=W \cup\{z\}$ with $z \notin W$. We will prove (3.5) for $V$, assuming that (3.5) is true for $W$ in place of $V$. Applying the assumption (3.7) to $V_{1}=W$ and $V_{2}=\{z\}$ to get the second equality below, the absorption and distributive laws to get the third, the induction hypothesis to get the fourth, and again the absorption and distributive laws in the fifth, we deduce that

$$
\begin{aligned}
a_{\emptyset}+ & a_{U_{1}} a_{U_{2}} b_{V} \\
& =a_{\emptyset}+a_{U_{1}} a_{U_{2}} b_{W \cup\{z\}} \\
& =a_{\emptyset}+a_{U_{1} \cap U_{2}} b_{W \cup\{z\}}+a_{U_{1}} a_{U_{2}}\left(b_{W}+b_{z}\right) \\
& =a_{\emptyset}+a_{U_{1} \cap U_{2}} b_{V}+\left(a_{\emptyset}+a_{U_{1}} a_{U_{2}} b_{W}\right)+a_{U_{1}} a_{U_{2}} b_{z} \\
& =a_{\emptyset}+a_{U_{1} \cap U_{2}} b_{V}+\left(a_{\emptyset}+a_{U_{1} \cap U_{2}} b_{W}+a_{U_{1}} a_{U_{2}}\left(b_{\emptyset}+\sum_{w \in W} b_{w}\right)\right)+a_{U_{1}} a_{U_{2}} b_{z} \\
& =a_{\emptyset}+a_{U_{1} \cap U_{2}} b_{V}+a_{U_{1}} a_{U_{2}}\left(b_{\emptyset}+\sum_{v \in W \cup\{z\}} b_{v}\right) \\
& =a_{\emptyset}+a_{U_{1} \cap U_{2}} b_{V}+a_{U_{1}} a_{U_{2}}\left(b_{\emptyset}+\sum_{v \in V} b_{v}\right)
\end{aligned}
$$

completing the proof.

To prepare the proof of the implication (iii) $\Rightarrow$ (i) in Theorem 3.5, we will show now that the equalities (3.5) and (3.6) extend to any finite number of $U_{i}$ 's and $V_{j}$ 's.

Lemma 3.8. Let $f, g \in \operatorname{PClo}(L)$ be as in Theorem 3.5. If $f$ and $g$ satisfy condition (iii) in Theorem 3.5, then they also satisfy the following condition:

(†) (a) $a_{\emptyset}+b_{\emptyset} \leq a_{[m]} b_{[n]}$ (i.e., $\left.\operatorname{im}(f) \cap \operatorname{im}(g) \neq \emptyset\right)$, and

(b) the equalities

$$
\begin{aligned}
& a_{\emptyset}+\left(\prod_{i=1}^{k} a_{U_{i}}\right) b_{V}=a_{\emptyset}+a_{\bigcap_{i=1}^{k} U_{i}} b_{V}+\left(\prod_{i=1}^{k} a_{U_{i}}\right)\left(b_{\emptyset}+\sum_{v \in V} b_{v}\right), \\
& b_{\emptyset}+\left(\prod_{j=1}^{k} b_{V_{j}}\right) a_{U}=b_{\emptyset}+b_{\bigcap_{j=1}^{k} V_{j}} a_{U}+\left(\prod_{j=1}^{k} b_{V_{j}}\right)\left(a_{\emptyset}+\sum_{u \in U} a_{u}\right) .
\end{aligned}
$$

hold for all $k \geq 1, U_{1}, \ldots, U_{k}, U \subseteq[m]$ and $V_{1}, \ldots, V_{k}, V \subseteq[n]$. 
Proof. Assume that condition (iii) in Theorem 3.5 holds for $f$ and $g$. Again, by symmetry, it suffices to prove equality (3.9). We will proceed by induction on $k$. Let $k \geq 1, U_{1}, \ldots, U_{k} \subseteq[m]$, and $V \subseteq[n]$. For $k=1$, condition (3.9) takes the form $a_{\emptyset}+a_{U_{1}} b_{V}=a_{\emptyset}+a_{U_{1}} b_{V}+a_{U_{1}}\left(b_{\emptyset}+\sum_{v \in V} b_{v}\right)$, which is clearly true, since $b_{\emptyset}+\sum_{v \in V} b_{v} \leq b_{V}$. For $k=2$, (3.9) coincides with the equality (3.5), which holds by assumption.

From now on let $k \geq 3$, and suppose that (3.9) is true for $k-1$ in place of $k$, that is,

$$
a_{\emptyset}+\left(\prod_{i=1}^{k-1} a_{U_{i}}\right) b_{V}=a_{\emptyset}+a_{\bigcap_{i=1}^{k-1} U_{i}} b_{V}+\left(\prod_{i=1}^{k-1} a_{U_{i}}\right)\left(b_{\emptyset}+\sum_{v \in V} b_{v}\right) .
$$

Taking the meet of both sides with $a_{U_{k}}$ and using the distributive law together with $a_{\emptyset} \leq a_{U_{k}}$ we see that

$$
a_{\emptyset}+\left(\prod_{i=1}^{k} a_{U_{i}}\right) b_{V}=a_{\emptyset}+a_{\bigcap_{i=1}^{k-1} U_{i}} a_{U_{k}} b_{V}+\left(\prod_{i=1}^{k} a_{U_{i}}\right)\left(b_{\emptyset}+\sum_{v \in V} b_{v}\right) .
$$

Applying the equality (3.5) to the sets $\bigcap_{i=1}^{k-1} U_{i}, U_{k}$, and $V$ on the right-hand side we obtain that

$$
\begin{aligned}
& a_{\emptyset}+\left(\prod_{i=1}^{k} a_{U_{i}}\right) b_{V} \\
& \quad=a_{\emptyset}+a_{\bigcap_{i=1}^{k} U_{i}} b_{V}+a_{\bigcap_{i=1}^{k-1} U_{i}} a_{U_{k}}\left(b_{\emptyset}+\sum_{v \in V} b_{v}\right)+\left(\prod_{i=1}^{k} a_{U_{i}}\right)\left(b_{\emptyset}+\sum_{v \in V} b_{v}\right) \\
& \quad=a_{\emptyset}+a_{\bigcap_{i=1}^{k} U_{i}} b_{V}+\left(\prod_{i=1}^{k} a_{U_{i}}\right)\left(b_{\emptyset}+\sum_{v \in V} b_{v}\right),
\end{aligned}
$$

where the last equality follows by observing that the monotonicity of the coefficients in a maximal DNF implies that $a_{\bigcap_{i=1}^{k-1} U_{i}} \leq \prod_{i=1}^{k-1} a_{U_{i}}$, and hence that the joinand $a_{\bigcap_{i=1}^{k-1} U_{i}} a_{U_{k}}\left(b_{\emptyset}+\sum_{v \in V} b_{v}\right)$ can be omitted. This completes the proof of Lemma 3.8.

Lemma 3.9. Let $f, g \in \mathrm{PClo}(L)$ be as in Theorem 3.5. If $f$ and $g$ satisfy condition ( $\ddagger$ ) in Lemma 3.8, then they also satisfy the equality (3.1) for all $R \subseteq[m] \times[n]$.

Proof. Assume that $f$ and $g$ satisfy condition $(\ddagger)$ in Lemma 3.8, and let $R \subseteq$ $[m] \times[n]$. Since the two sides of (3.1) can be obtained from one another by switching the roles of the coefficients $a_{S}$ of $f$ with the coefficients $b_{T}$ of $g$, and since the assumption $(\ddagger)$ is invariant under this switch, it will be sufficient to verify the inequality $\geq$ in (3.1). To start, we notice that for the choice $S=\emptyset$ the joinand $a_{S} \prod_{i \in S} b_{R(i,-)}$ on the left-hand side of (3.1) is equal to $a_{\emptyset}$, while for the choice $S=[m]$ it is $\geq a_{[m]} b_{\emptyset}$. Therefore, to verify $\geq$ in (3.1) it suffices to show that for every set $T \subseteq[n]$,

$$
a_{\emptyset}+a_{[m]} b_{\emptyset}+\sum_{S \subseteq[m]} a_{S} \prod_{i \in S} b_{R(i,-)} \geq a_{\emptyset}+\left(\prod_{j \in T} a_{R(-, j)}\right) b_{T} .
$$

If $T=\emptyset$, then $a_{\emptyset}+\left(\prod_{j \in T} a_{R(-, j)}\right) b_{T}=a_{\emptyset}+b_{\emptyset}=a_{\emptyset}+a_{[m]} b_{\emptyset}$ by Lemma 3.3 , so (3.11) holds in this case. From now on we will assume that $T \neq \emptyset$, and set 
$\hat{S}=\bigcap_{j \in T} R(-, j)$. Thus, applying (3.9) to the right-hand side of (3.11), simplifying the last joinand by taking into account that $b_{\emptyset}+\sum_{t \in T} b_{t}=\sum_{t \in T} b_{t}$ if $T \neq \emptyset$, and then using the distributive law, we get that

$$
\begin{aligned}
a_{\emptyset}+\left(\prod_{j \in T} a_{R(-, j)}\right) b_{T} & =a_{\emptyset}+a_{\bigcap_{j \in T}} R(-, j) \\
& b_{T}+\left(\prod_{j \in T} a_{R(-, j)}\right) \sum_{t \in T} b_{t} \\
& =a_{\emptyset}+a_{\hat{S}} b_{T}+\sum_{t \in T}\left(\prod_{j \in T} a_{R(-, j)}\right) b_{t} .
\end{aligned}
$$

Here $a_{\hat{S}} b_{T} \leq a_{\hat{S}} \prod_{i \in \hat{S}} b_{R(i,-)}$, because $\hat{S} \subseteq R(-, j)$ for all $j \in T$ implies that $\hat{S} \times T \subseteq R$, whence it follows that $T \subseteq R(i,-)$ and $b_{T} \leq b_{R(i,-)}$ hold for all $i \in \hat{S}$. Thus $a_{\hat{S}} b_{T}$ is bounded above by one of the joinands on the left-hand side of (3.11). Similarly, for each other joinand $\left(\prod_{j \in T} a_{R(-, j)}\right) b_{t}$ in (3.12) where $t \in T$, we have that $\left(\prod_{j \in T} a_{R(-, j)}\right) b_{t} \leq a_{R(-, t)} b_{t}$, and $a_{R(-, t)} b_{t}$ is bounded above by one of the joinands on the left-hand side of (3.11) for the following reason: $a_{R(-, t)} b_{t} \leq a_{R(-, t)} \prod_{i \in R(-, t)} b_{R(i,-)}$, because $t \in R(i,-)$ for all $i \in R(-, t)$.

This proves (3.11), and therefore completes the proof of Lemma 3.9.

Now we are ready to complete the proof of Theorem 3.5.

Proof of Theorem 3.5. The implication (i) $\Rightarrow$ (ii) was established in Lemma 3.4, while the implication (ii) $\Rightarrow$ (iii) follows from Lemmas 3.6 and 3.7. Finally, Lemmas 3.8 and 3.9 , combined with Proposition 3.1 , show that (iii) $\Rightarrow$ (i).

Corollary 3.10. Let $L$ be a distributive lattice, and let $f, g$ be polynomial operations of $L$ with maximal DNFs

$$
f\left(x_{1}, \ldots, x_{m}\right)=\sum_{S \subseteq[m]} a_{S} \prod_{i \in S} x_{i} \quad \text { and } \quad g\left(x_{1}, \ldots, x_{n}\right)=\sum_{T \subseteq[n]} b_{T} \prod_{j \in T} x_{j} .
$$

In addition to conditions (ii)-(iii) in Theorem 3.5 the conditions listed below are also equivalent to $f \perp g$ :

(iv) condition ( $\ddagger$ ) in Lemma 3.8, which strengthens (iii);

(v) condition $(\dagger)_{2}$ in Lemma 3.6;

(vi) the following conditions, which strengthen (v):

(a) $a_{\emptyset}+b_{\emptyset} \leq a_{[m]} b_{[n]}$ (i.e., $\operatorname{im}(f) \cap \operatorname{im}(g) \neq \emptyset$ ), and

(b) the equalities

$$
\begin{gathered}
a_{\emptyset}+\left(\prod_{i=1}^{k} a_{U_{i}}\right) b_{\bigcup_{j=1}^{\ell} V_{j}}=a_{\emptyset}+a_{\bigcap_{i=1}^{k} U_{i}} b_{\bigcup_{j=1}^{\ell} V_{j}}+\left(\prod_{i=1}^{k} a_{U_{i}}\right) \sum_{j=1}^{\ell} b_{V_{j}}, \\
b_{\emptyset}+\left(\prod_{j=1}^{\ell} b_{V_{j}}\right) a_{\bigcup_{i=1}^{k} U_{i}}=b_{\emptyset}+b_{\bigcap_{j=1}^{\ell} V_{j}} a_{\bigcup_{i=1}^{k} U_{i}}+\left(\prod_{j=1}^{\ell} b_{V_{j}}\right) \sum_{i=1}^{k} a_{U_{i}} \\
\quad \text { hold for all } k, \ell \geq 1, U_{1}, \ldots, U_{k} \subseteq[m] \text { and } V_{1}, \ldots, V_{\ell} \subseteq[n] .
\end{gathered}
$$

Proof. Theorem 3.5 was proved via the implications $f \perp g \Rightarrow$ (ii) $\Rightarrow$ (v) $\Rightarrow$ (iii) $\Rightarrow$ (iv) $\Rightarrow f \perp g$, so conditions (ii)-(v) are all equivalent to $f \perp g$. Clearly, (v) is the special case $k=2=\ell$ of (vi), therefore (vi) $\Rightarrow$ (v). Finally, we show that 
(iv) $\Rightarrow$ (vi). To this end we have to prove that (3.9)-(3.10) imply (3.13)-(3.14). If $V=\bigcup_{j=1}^{\ell} V_{j}$, then by comparing the equality (3.13) with the equality (3.9) we see that (3.13) and (3.9) have the same left-hand sides, while the right-hand side of (3.13) is less than or equal to the common left-hand sides of (3.9) and (3.13), but is greater than or equal to the right-hand side of (3.9). Thus, (3.9) implies (3.13), and similarly, (3.10) implies (3.14). This proves that condition (vi) is equivalent to (ii)-(v), and hence to $f \perp g$.

\section{Applications}

4.1. Self-commuting lattice polynomial operations. Let $L$ be a distributive lattice, and let $f, g$ be polynomial operations of $L$. Applying the characterizations of $f \perp g$ in Theorem 3.5 and Corollary 3.10 to the case when $f=g$, we can obtain analogous characterizations of self-commuting polynomial operations of distributive lattices. The conditions obtained in this way can be simplified by observing that the requirement $\operatorname{im}(f) \cap \operatorname{im}(g)=\emptyset$ holds automatically for $f=g$. Moreover, in the remaining requirements the joinands $a_{\emptyset}=b_{\emptyset}$ on both sides of the equalities can be omitted, since they are dominated by the remaining joinands on both sides. In the corollary below we will state only the characterizations obtained from Theorem 3.5.

Corollary 4.1. Let $L$ be a distributive lattice, and let $f$ be a polynomial operation of $L$ with maximal DNF

$$
f\left(x_{1}, \ldots, x_{m}\right)=\sum_{S \subseteq[m]} a_{S} \prod_{i \in S} x_{i} .
$$

The following conditions on $f$ equivalent:

(i) $f \perp f$;

(ii) the equality

$$
\begin{aligned}
& a_{U_{1} \cap U_{2}} a_{V_{1} \cup V_{2}}+a_{U_{1}} a_{V_{1}}+a_{U_{2}} a_{V_{2}}+a_{U_{1} \cup U_{2}} a_{V_{1}} a_{V_{2}} \\
& \quad=a_{U_{1}} a_{U_{2}} a_{V_{1} \cup V_{2}}+a_{U_{1}} a_{V_{1}}+a_{U_{2}} a_{V_{2}}+a_{U_{1} \cup U_{2}} a_{V_{1} \cap V_{2}}
\end{aligned}
$$

holds for all $U_{1}, U_{2}, V_{1}, V_{2} \subseteq[m]$;

(iii) the equality

$$
a_{U_{1}} a_{U_{2}} a_{V}=a_{U_{1} \cap U_{2}} a_{V}+a_{U_{1}} a_{U_{2}}\left(a_{\emptyset}+\sum_{v \in V} a_{v}\right)
$$

holds for all $U_{1}, U_{2}, V \subseteq[m]$.

Next we will apply Corollary 4.1 to obtain the main result of [3], which is an explicit description of all self-commuting polynomial operations of a bounded chain. We will state the result for a wider class of polynomial operations, but in view of Corollary 3.2 this is equivalent to the original formulation.

Corollary 4.2. Let $L$ be a distributive lattice. If $f$ is a polynomial operation of $L$ with a DNF

$$
f\left(x_{1}, \ldots, x_{m}\right)=\sum_{S \subseteq[m]} \tilde{a}_{S} \prod_{i \in S} x_{i}
$$

such that the set $\left\{\tilde{a}_{S}: S \subseteq[m]\right\}$ of coefficients is a chain in $L^{01}$, then the following conditions on $f$ are equivalent:

(i) $f \perp f$; 
(ii) $f$ has a $D N F$

$$
f\left(x_{1}, \ldots, x_{m}\right)=a_{\emptyset}+\sum_{i \in[m]} a_{i} x_{i}+\sum_{\ell=1}^{r} a_{S_{\ell}} \prod_{i \in S_{\ell}} x_{i}
$$

such that

(1) $S_{1} \subset S_{2} \subset \cdots \subset S_{r} \subseteq[m](r \geq 0)$,

(2) $\left\{a_{\emptyset}\right\} \cup\left\{a_{i}: i \in[m]\right\}$ is a chain, and

$$
a_{\emptyset}+\sum_{i \in S_{1}} a_{i}=a_{\emptyset}+\sum_{i \in[m]} a_{i}<a_{S_{1}}<\cdots<a_{S_{r}} .
$$

Remark 4.3. Condition (ii) is stated here in a slightly different form than in [3], but the two formulations are equivalent.

Proof of Corollary 4.2. To prove the implication (i) $\Rightarrow$ (ii) assume that $f \perp f$, and let

$$
f\left(x_{1}, \ldots, x_{m}\right)=\sum_{S \subseteq[m]} a_{S} \prod_{i \in S} x_{i}
$$

be the maximal DNF of $f$. By the definition of maximal DNF, the coefficients $a_{S} \in L^{01}$ satisfy $a_{S} \leq a_{T}$ whenever $S \subseteq T \subseteq[m]$. We will use this property without further reference. In addition, since $f \perp f$, the coefficients also satisfy (4.2) for all $U_{1}, U_{2}, V \subseteq[m]$. Notice also that by Proposition 2.2 the coefficients $a_{U}$ are obtained from the coefficients $\tilde{a}_{S}$ of the given DNF by taking joins. Therefore, the hypothesis that $\left\{\tilde{a}_{S}: S \subseteq[m]\right\}$ is a chain in $L^{01}$ implies that $\left\{a_{U}: U \subseteq[m]\right\}$ is also a chain in $L^{01}$.

Let $\mathcal{E}$ denote the set of all $S \subseteq[\mathrm{m}]$ such that $|S| \geq 2$ and the $S$-term of (4.4) is essential, i.e., $a_{S}>\sum_{U \subset S} a_{U}$. First we will prove that

$$
a_{S} \leq a_{T} \Longleftrightarrow S \subseteq T \quad \text { for all } S, T \in \mathcal{E} .
$$

Since the implication $\Leftarrow$ is clear, suppose for a contradiction that $\Rightarrow$ is false, that is, for some $S, T \in \mathcal{E}$ we have $a_{S} \leq a_{T}$ but $S \nsubseteq T$. Then $S \cap T \subset S$, and hence the fact that $a_{S}$ is essential implies that $a_{S \cap T}<a_{S}$. Now, applying (4.2) to $U_{1}=S$, $U_{2}=T$, and $V=S$ we get that

$$
a_{S} a_{T} a_{S}=a_{S \cap T} a_{S}+a_{S} a_{T}\left(a_{\emptyset}+\sum_{s \in S} a_{s}\right) .
$$

Since $a_{\emptyset}+\sum_{s \in S} a_{s} \leq a_{S}$, and as we have seen, $a_{S \cap T}<a_{S} \leq a_{T}$, therefore the displayed equality simplifies to $a_{S}=a_{S \cap T}+\left(a_{\emptyset}+\sum_{s \in S} a_{s}\right)=a_{\emptyset}+\sum_{s \in S} a_{s}+a_{S \cap T}$. Since $|S| \geq 2$ and $S \cap T \subset S$, this equality shows that, contrary to the choice of $S$, the $S$-term of (4.4) is inessential. This proves (4.5).

Since $\left\{a_{U}: U \subseteq[m]\right\}$ is a chain, (4.5) implies that $\mathcal{E}$ is a chain of subsets of $[m]$, say, $\mathcal{E}=\left\{S_{\ell}: 1 \leq \ell \leq r\right\}$ with $S_{1} \subset S_{2} \subset \cdots \subset S_{r}(r \geq 0)$. Thus, (1) and the inequalities $a_{\emptyset}+\sum_{i \in S_{1}} a_{i}<a_{S_{1}}<\cdots<a_{S_{r}}$ from (2) are true. Moreover, since $\mathcal{E}$ contains all $S \subseteq[m]$ with $|S| \geq 2$ for which the $S$-term of (4.4) is essential, (4.3) also holds. The condition from (2) that $\left\{a_{\emptyset}\right\} \cup\left\{a_{i}: i \in[m]\right\}$ is a chain is obviously satisfied.

Therefore, it remains to show the equality $a_{\emptyset}+\sum_{i \in S_{1}} a_{i}=a_{\emptyset}+\sum_{i \in[m]} a_{i}$ from (2). Suppose that the equality fails, that is, $a_{\emptyset}+\sum_{i \in S_{1}} a_{i}<a_{\emptyset}+\sum_{i \in[m]} a_{i}$. The fact that $\left\{a_{\emptyset}\right\} \cup\left\{a_{i}: i \in[m]\right\}$ is a chain implies then that $a_{\emptyset}+\sum_{i \in[m]} a_{i}=a_{p}$ 
for some $p \in[m]$, and $p \notin S_{1}$. Thus, applying (4.2) to $U_{1}=S_{1}, U_{2}=\{p\}$, and $V=S_{1}$ we get that

$$
a_{S_{1}} a_{\{p\}} a_{S_{1}}=a_{S_{1} \cap\{p\}} a_{S_{1}}+a_{S_{1}} a_{\{p\}}\left(a_{\emptyset}+\sum_{s \in S_{1}} a_{s}\right) .
$$

The first joinand on the right-hand side can be omitted, since $S_{1} \cap\{p\}=\emptyset$. Furthermore, $a_{\emptyset}+\sum_{s \in S_{1}} a_{s}<a_{p}$ by the choice of $a_{p}$, and $a_{\emptyset}+\sum_{s \in S_{1}} a_{s}<a_{S_{1}}$, since $S_{1} \in \mathcal{E}$. Since $a_{S_{1}}, a_{p}$ are comparable, this implies that $a_{\emptyset}+\sum_{s \in S_{1}} a_{s}<a_{S_{1}} a_{p}$. Thus the displayed equality simplifies to $a_{S_{1}} a_{p}=a_{\emptyset}+\sum_{s \in S_{1}} a_{s}$, contradicting the preceding conclusion. This completes the proof of (i) $\Rightarrow$ (ii).

For the reverse implication (ii) $\Rightarrow$ (i) let us assume that (ii) holds. Condition (ii) remains valid if we replace each coefficient $a_{i}(i \in[m])$ with $a_{\emptyset}+a_{i}$, therefore we may assume without loss of generality that $a_{\emptyset} \leq a_{i}$ holds for all $i \in[m]$. Under this additional assumption one can easily see, using Proposition 2.2, that for each one of the sets $S=\emptyset, S=\{i\}$ with $i \in[m]$, and $S=S_{\ell}$ with $1 \leq \ell \leq r$, the $S$-coefficient of the maximal DNF of $f$ is $a_{S}$. Therefore, we can describe all coefficients of the maximal DNF of $f$ as follows:

$$
a_{S}= \begin{cases}a_{S_{\ell}} & \text { if } \left.S_{\ell} \subseteq S \text { and } S_{\ell+1} \nsubseteq S(1 \leq \ell \leq)\right), \\ a_{\emptyset}+\sum_{s \in S} a_{s} & \text { if } S_{1} \nsubseteq S .\end{cases}
$$

In view of Corollary 4.1, the proof of (i) will be complete if we verify that (4.2) holds for all $U_{1}, U_{2}, V \subseteq[m]$.

Since the inequality $\geq$ is clearly true in (4.2), we will prove $=$ by showing that the element on left-hand side of (4.2) is dominated by one of the joinands on the right-hand side of (4.2). If $S_{1} \nsubseteq \subseteq V$, i.e., $a_{V}=a_{\emptyset}+\sum_{v \in V} a_{v}$, then this is obviously the case. Therefore, we will assume from now on that $S_{1} \subseteq V$. Let $\ell$ be such that $S_{\ell} \subseteq V$, but $S_{\ell+1} \nsubseteq V$. Thus $a_{V}=a_{S_{\ell}}$. If one of $U_{1}, U_{2}$ fails to contain $S_{1}$, say, $S_{1} \nsubseteq U_{1}$, then

$$
a_{U_{1}}=a_{\emptyset}+\sum_{u \in U_{1}} a_{u} \leq a_{\emptyset}+\sum_{i \in[m]} a_{i}=a_{\emptyset}+\sum_{s \in S_{1}} a_{s} \leq a_{\emptyset}+\sum_{v \in V} a_{v} \leq a_{V}
$$

where the second $=$ follows from condition (2) in (ii), and the succeeding $\leq$ follows from $S_{1} \subseteq V$. Hence $a_{U_{1}} a_{U_{2}} a_{V}=a_{U_{1}} a_{U_{2}}=a_{U_{1}} a_{U_{2}} a_{U_{1}} \leq a_{U_{1}} a_{U_{2}}\left(a_{\emptyset}+\sum_{v \in V} a_{v}\right)$, completing the proof in this case. Finally, let $S_{1} \subseteq U_{1}, U_{2}$, say $S_{i} \subseteq U_{1}, S_{i+1} \nsubseteq U_{1}$, and $S_{j} \subseteq U_{2}, S_{j+1} \nsubseteq U_{2}$. We may assume without loss of generality that $i \leq j$. Then $S_{i} \subseteq U_{1} \cap U_{2}, S_{i+1} \nsubseteq U_{1} \cap U_{2}$. so $a_{U_{1}} a_{U_{2}} a_{V}=a_{S_{i}} a_{V}=a_{U_{1} \cap U_{2}} a_{V}$, which completes the proof of Corollary 4.2.

An $m$-ary operation $f$ is called symmetric, if it satisfies the identity

$$
f\left(x_{1}, x_{2}, \ldots, x_{m}\right)=f\left(x_{\sigma(1)}, x_{\sigma(2)}, \ldots, x_{\sigma(m)}\right)
$$

for all permutations $\sigma$ of $[\mathrm{m}]$. If $f$ is a polynomial operation of a distributive lattice $L$ with maximal DNF

$$
f\left(x_{1}, \ldots, x_{m}\right)=\sum_{S \subseteq[m]} a_{S} \prod_{i \in S} x_{i}
$$

then the uniqueness of maximal DNFs (see Proposition 2.2) implies that $f$ is symmetric if and only if $a_{I}=a_{J}$ whenever $|I|=|J|(I, J \subseteq[m])$. It follows that in this case $\left\{a_{S}: S \subseteq[m]\right\}$ is a chain in $L^{01}$. Hence we can apply Corollary 4.2 
to characterize when a symmetric polynomial operation of a distributive lattice is self-commuting.

Corollary 4.4. A polynomial operation $f$ of a distributive lattice is symmetric and self-commuting if and only if it has a DNF of the form

$$
f\left(x_{1}, \ldots, x_{m}\right)=a_{\emptyset}+\sum_{i \in[m]} a_{1} x_{i}+a_{[m]} \prod_{i \in[m]} x_{i}
$$

for some $a_{\emptyset}, a_{1}, a_{[m]} \in L^{01}$ with $a_{\emptyset} \leq a_{1} \leq a_{[m]}$.

Proof. If $f$ has such a DNF, then $f$ is symmetric. Furthermore, if $a_{1}<a_{[m]}$, then the given DNF satisfies condition (ii) in Corollary 4.2 with $r=1$, while if $a_{1}=a_{[m]}$, then the $[m]$-term of the given DNF is inessential and can be omitted, so the resulting DNF satisfies condition (ii) in Corollary 4.2 with $r=0$. In either case, $f$ is self-commuting.

Conversely, assume that $f$ is symmetric and self-commuting. As we observed above, symmetry implies that in the maximal DNF (4.6) of $f$ we have $a_{I}=a_{J}$ whenever $|I|=|J|(I, J \subseteq[m])$. Now, if an $I$-term in (4.6) is essential, then so are all $J$-terms with $|J|=|I|$. However, as we have seen in the proof of (ii) $\Rightarrow$ (i) in Corollary 4.2, if $f$ is self-commuting, then the sets $S \subseteq[m]$ with $|S| \geq 2$ for which the $S$-terms of the maximal DNF of $f$ are essential form a chain. This forces that the only set $S$ with $|S| \geq 2$ for which the $S$-term in (4.6) may be essential is $S=[m]$. Thus an $S$-term in (4.6) is essential only if $|S| \in\{0,1, m\}$, so $f$ has the prescribed form.

4.2. Commutativity of special lattice polynomial operations. We will now use our theorem on commuting pairs of distributive lattice polynomial operations to determine all commuting pairs of distributive lattice term operations. At the end of this subsection we will outline a second proof of the same result that does not use our theorem on commuting polynomials.

When determining pairs of commuting term operations, one special case that is key to the general argument is the case where one term is a join of two variables. We shall work out that case first in a bit more generality than necessary, namely we will describe those polynomial operations of a distributive lattice which commute with an arbitrary linear polynomial operation. It seems plausible that this case will find application some day.

By a linear polynomial operation of a distributive lattice we mean a polynomial operation of the form

$$
f\left(x_{1}, \ldots, x_{m}\right)=a_{\emptyset}+\sum_{i \in[m]} a_{i} x_{i} \quad \text { where } m \geq 1 \text { and } a_{\emptyset} \leq a_{i} \text { for all } i \in[m] .
$$

For $1 \leq i<j \leq m$ let $\phi_{f}^{i j}$ denote the unary polynomial operation

$$
\phi_{f}^{i j}(x):=a_{\emptyset}+a_{i} a_{j} x
$$

of $L$. As $\phi_{f}^{i j}$ is a unary polynomial, it is a lattice homomorphism $L^{01} \rightarrow L^{01}$.

Corollary 4.5. Let $L$ be a distributive lattice, and let $f$ be a linear polynomial operation of $L$. A polynomial operation $g$ of $L$ with maximal DNF $g\left(x_{1}, \ldots, x_{n}\right)=$ $\sum_{T \subseteq[n]} b_{T} \prod_{j \in T} x_{j}$ commutes with $f$ if and only if

(a) $a_{\emptyset}+b_{\emptyset} \leq\left(\sum_{i \in[m]} a_{i}\right) b_{[n]}($ i.e., $\operatorname{im}(f) \cap \operatorname{im}(g) \neq \emptyset)$, and 
(a) $\phi_{f}^{i j}\left(b_{V}\right)=\phi_{f}^{i j}\left(b_{\emptyset}+\sum_{v \in V} b_{v}\right)$ for all $V \subseteq[n]$ and $1 \leq i<j \leq m$.

Since $\phi_{f}^{i j}: L^{01} \rightarrow L^{01}$ are lattice homomorphisms, condition (b) here is easily seen to be equivalent to

(b) $\phi_{f}^{i j}\left(b_{V_{1} \cup V_{2}}\right)=\phi_{f}^{i j}\left(b_{V_{1}}+b_{V_{2}}\right)$ for all $V_{1}, V_{2} \subseteq[n]$ and $1 \leq i<j \leq m$.

Proof of Corollary 4.5. The assumption $a_{\emptyset} \leq a_{i}$ for all $i \in[m]$ ensures that the coefficients $a_{U}(U \subseteq[m])$ of the maximal DNF of $f$ are $a_{U}=a_{\emptyset}+\sum_{u \in U} a_{u}$ for all $U \subseteq[m]$. By Theorem 3.5, $f \perp g$ if and only if (a) holds and the equalities (3.5)-(3.6) are satisfied by the coefficients $a_{U}, b_{V}$ of the maximal DNFs of $f$ and $g$. For our $f$, the equality (3.6) holds automatically for arbitrary $U \subseteq[m]$ and $V_{1}, V_{2} \subseteq[n]$. Now let $U_{1}, U_{2} \subseteq[m]$ and $V \subseteq[n]$. Since

$$
\begin{aligned}
a_{U_{1}} a_{U_{2}} & =\left(a_{\emptyset}+\sum_{u_{1} \in U_{1}} a_{u_{1}}\right)\left(a_{\emptyset}+\sum_{u_{2} \in U_{2}} a_{u_{2}}\right) \\
& =a_{\emptyset}+\sum_{u \in U_{1} \cap U_{2}} a_{u}+\sum_{\substack{u_{1} \in U_{1} \backslash U_{2} \\
u_{2} \in U_{2} \backslash U_{1}}} a_{u_{1}} a_{u_{2}}=a_{U_{1} \cap U_{2}}+\sum_{\substack{u_{1} \in U_{1} \backslash U_{2} \\
u_{2} \in U_{2} \backslash U_{1}}} a_{u_{1}} a_{u_{2}},
\end{aligned}
$$

the equality (3.5) is equivalent to the following:

$$
\begin{gathered}
a_{\emptyset}+a_{U_{1} \cap U_{2}} b_{V}+\left(\sum_{\substack{u_{1} \in U_{1} \backslash U_{2} \\
u_{2} \in U_{2} \backslash U_{1}}} a_{u_{1}} a_{u_{2}}\right) b_{V} \\
\quad=a_{\emptyset}+a_{U_{1} \cap U_{2}} b_{V}+\left(\sum_{\substack{u_{1} \in U_{1} \backslash U_{2} \\
u_{2} \in U_{2} \backslash U_{1}}} a_{u_{1}} a_{u_{2}}\right)\left(b_{\emptyset}+\sum_{v \in V} b_{v}\right) .
\end{gathered}
$$

In the special case when $U_{1}=\{i\}$ and $U_{2}=\{j\}(1 \leq i<j \leq m)$ we have $a_{U_{1} \cap U_{2}}=a_{\emptyset}$, so (4.8) simplifies to

$$
a_{\emptyset}+a_{i} a_{j} b_{V}=a_{\emptyset}+a_{i} a_{j}\left(b_{\emptyset}+\sum_{v \in V} b_{v}\right),
$$

i.e., $\phi_{f}^{i j}\left(b_{V}\right)=\phi_{f}^{i j}\left(b_{\emptyset}+\sum_{v \in V} b_{v}\right)$. Conversely, the equality (4.8) can be obtained by joining to the obvious equality $a_{\emptyset}+a_{U_{1} \cap U_{2}} b_{V}=a_{\emptyset}+a_{U_{1} \cap U_{2}} b_{V}$ all equalities (4.9) where $\{i, j\}=\left\{u_{1}, u_{2}\right\}, u_{1} \in U_{1} \backslash U_{2}, u_{2} \in U_{2} \backslash U_{1}$, and $1 \leq i<j \leq m$. This proves Corollary 4.5 .

The dual statement to Corollary 4.5 concerns polynomial operations $f$ of the form

$$
\begin{array}{r}
f\left(x_{1}, \ldots, x_{m}\right)=a_{[m]} \prod_{i \in[m]}\left(a_{[m] \backslash\{i\}}+x_{i}\right)=\sum_{S \subseteq[m]}\left(a_{[m]} \prod_{i \notin S} a_{[m] \backslash\{i\}}\right) \prod_{i \in S} x_{i} \\
\text { where } m \geq 1 \text { and } a_{[m]} \geq a_{[m] \backslash\{i\}} \text { for all } i \in[m] .
\end{array}
$$

For $1 \leq i<j \leq m$ let now $\psi_{f}^{i j}$ denote the unary polynomial operation

$$
\psi_{f}^{i j}(x):=a_{[m]}\left(a_{[m] \backslash\{i\}}+a_{[m] \backslash\{j\}}+x\right)=a_{[m] \backslash\{i\}}+a_{[m] \backslash\{j\}}+a_{[m]} x
$$

of $L$. Again, $\psi_{f}^{i j}$ is a lattice homomorphism $L^{01} \rightarrow L^{01}$. 
Corollary 4.6. Let $L$ be a distributive lattice, and let $f$ be a polynomial operation of $L$ satisfying (4.10). A polynomial operation $g$ of $L$ with maximal DNF $g\left(x_{1}, \ldots, x_{n}\right)=\sum_{T \subseteq[n]} b_{T} \prod_{j \in T} x_{j}$ commutes with $f$ if and only if

(a) $\left(\prod_{i \in[m]} a_{[m] \backslash\{i\}}\right)+b_{\emptyset} \leq a_{[m]} b_{[n]}$ (i.e., $\left.\operatorname{im}(f) \cap \operatorname{im}(g) \neq \emptyset\right)$, and

(b) $\psi_{f}^{i j}\left(b_{V}\right)=\psi_{f}^{i j}\left(b_{[n]} \prod_{v \notin V} b_{[n] \backslash\{v\}}\right)$ for all $V \subseteq[n]$ and $1 \leq i<j \leq m$.

Since $\psi_{f}^{i j}: L^{01} \rightarrow L^{01}$ are lattice homomorphisms, condition (b) here is easily seen to be equivalent to

(b) ${ }^{\prime} \psi_{f}^{i j}\left(b_{V_{1} \cap V_{2}}\right)=\psi_{f}^{i j}\left(b_{V_{1}} b_{V_{2}}\right)$ for all $V_{1}, V_{2} \subseteq[n]$ and $1 \leq i<j \leq m$.

Now we are ready to determine all pairs of commuting term operations of a distributive lattice.

Corollary 4.7. Let $L$ be a distributive lattice. Two term operations $f$ and $g$ of $L$ commute if and only if they satisfy one of the following conditions:

(a) one of $f, g$ is a projection, and the other one is arbitrary,

(b) both of $f$ and $g$ are joins of variables,

(c) both of $f$ and $g$ are meets of variables.

Proof. The sufficiency of the given condition for $f \perp g$ is clear. To prove the necessity assume that $f \perp g$ and neither $f$ nor $g$ is a projection. Let

$$
f\left(x_{1}, \ldots, x_{m}\right)=\sum_{S \subseteq[m]} a_{S} \prod_{i \in S} x_{i} \quad \text { and } \quad g\left(x_{1}, \ldots, x_{n}\right)=\sum_{T \subseteq[n]} b_{T} \prod_{j \in T} x_{j},
$$

be the maximal DNFs of $f$ and $g$. Since $f$ and $g$ are term operations, $a_{S}, b_{T} \in\{0,1\}$ for all $S \subseteq[m]$ and $T \subseteq[n]$. Let

$$
\mathcal{F}:=\left\{S \subseteq[m]: a_{S}=1\right\} \quad \text { and } \quad \mathcal{G}:=\left\{T \subseteq[n]: b_{T}=1\right\} .
$$

$\mathcal{F}$ is an order filter in the power set of $[m]$, and $\mathcal{G}$ is an order filter in the power set of $[n]$. Moreover, $a_{\emptyset}=b_{\emptyset}=0$ and $a_{[m]}=b_{[n]}=1$; that is, $\emptyset \notin \mathcal{F},[m] \in \mathcal{F}$, and $\emptyset \notin \mathcal{G},[n] \in \mathcal{G}$.

We will distinguish two cases. Suppose first that $\mathcal{F}$ has a least element, $S_{0}$. Then $S_{0} \neq \emptyset$ and the $S_{0}$-term is the only essential term of the maximal DNF of $f$, so $f\left(x_{1}, \ldots, x_{m}\right)=\prod_{i \in S_{0}} x_{i}$. By assumption, $f$ is not a projection, therefore $\left|S_{0}\right| \geq 2$. Since $f$ generates the same clone as $x_{1} x_{2}$, we have $f \perp g$ if and only if $x_{1} x_{2} \perp g$. Applying Corollary 4.6 with $m=2$ and $a_{[2]}=1, a_{1}=a_{2}=0$, we see that $\psi^{12}$ is the identity homomorphism, and hence $\mathcal{G}$ is closed under $\cap$ by Corollary $4.6(\mathrm{~b})^{\prime}$. The intersection of all elements of $\mathcal{G}$ yields a least element $T_{0}$ of $\mathcal{G}$, and $g\left(x_{1}, \ldots, x_{n}\right)=\prod_{j \in T_{0}} x_{j}$. This shows that if $f \perp g$ and $f$ is a meet of at least two variables, then $g$ is also a meet of variables.

It remains to consider the case when $\mathcal{F}$ has two incomparable minimal elements, say $U_{1}$ and $U_{2}$. In this case $a_{U_{1}}=a_{U_{2}}=1$, but $a_{U_{1} \cap U_{2}}=0$, while we still have $a_{\emptyset}=b_{\emptyset}=0$. We will substitute these values into condition (3.5) of Theorem 3.5, which we recall here:

$$
a_{\emptyset}+a_{U_{1}} a_{U_{2}} b_{V}=a_{\emptyset}+a_{U_{1} \cap U_{2}} b_{V}+a_{U_{1}} a_{U_{2}}\left(b_{\emptyset}+\sum_{v \in V} b_{v}\right) .
$$

The result of the substitution is that $b_{V}=\sum_{v \in V} b_{v}$ holds for arbitrary $V \subseteq[n]$, which shows that if a $V$-term of $g$ is essential, then $V$ is a singleton. Hence 
$g\left(x_{1}, \ldots, x_{n}\right)=\sum_{t \in T_{0}} x_{t}$ for some $T_{0} \subseteq[n]$. By assumption, $g$ is not a projection, so it is a join of at least two variables. By the dual of the last sentence of the preceding paragraph, $f$ must also be a join of variables.

Now we outline a second proof of Corollary 4.7. Suppose that $f$ and $g$ are term operations of a nontrivial distributive lattice $L$. The restriction map to a 2 -element sublattice of $L$ is a clone isomorphism, because the variety of distributive lattices is minimal, so $f$ and $g$ commute on $L$ if and only if they commute on some (any) 2-element sublattice. Thus no generality is lost in assuming that $L=\{0,1\}$ has only two elements.

Let $\mathcal{I}$ denote the clone of all idempotent operations on $\{0,1\}$. Let $\mathcal{C}$ be the clone generated by $f$, let $\mathcal{D}=\mathcal{C}^{\perp} \cap \mathcal{I}$ be the clone of idempotent operations centralizing $\mathcal{C}$, and let $\mathcal{E}=\mathcal{D}^{\perp} \cap \mathcal{I}$ be the clone of idempotent operations centralizing $\mathcal{D}$. We have that $\{\mathcal{D}, \mathcal{E}\}$ is an unordered pair of idempotent clones, each the "idempotent centralizer" of the other, and that $g \in \mathcal{D}$ and $f \in \mathcal{E}$. By examining Post's lattice of all clones on the 2 -element set [12] and by determining the centralizer clones among them $[7,10]$ it is easy to see that there are four such pairs $\{\mathcal{D}, \mathcal{E}\}$ :

(1) $\{\mathcal{D}, \mathcal{E}\}=\{$ the clone of projections, $\mathcal{I}\}$,

(2) $\mathcal{D}=\mathcal{E}=$ the clone generated by ternary addition modulo 2 ,

(3) $\mathcal{D}=\mathcal{E}=$ the clone generated by meet, or

(4) $\mathcal{D}=\mathcal{E}=$ the clone generated by join.

In case (1), one of $f$ or $g$ must be a projection. In case (2), both $f$ and $g$ are monotone affine operations, hence again must be projections. So if neither $f$ nor $g$ is a projection, then both are nonprojections from the same (minimal) clone generated by one of the semilattice operations. This forces them both to be meets of variables or both to be joins of variables.

\section{REFERENCES}

[1] J. AczéL, J. Dhombres, Functional Equations in Several Variables, Encyclopedia of Mathematics and Its Applications, vol. 31, Cambridge University Press, Cambridge, 1989.

[2] S. Burris, R. Willard, Finitely many primitive positive clones, Proc. Amer. Math. Soc. 101 (1987), no. 3, 427-430.

[3] M. Couceiro, E. Lehtonen, Self-commuting lattice polynomial functions, arXiv:0912.0478.

[4] G. Eigenthaler, R. Winkler, Commutative composition semigroups of polynomials, Contributions to General Algebra 6, Hölder-Pichler-Tempsky, Vienna, 1988, pp. 89-101.

[5] R. L. Goodstein, The solution of equations in a lattice, Proc. Roy. Soc. Edinburgh Sect. A $67(1965 / 1967), 231-242$.

[6] M. Grabisch, J.-L. Marichal, R. Mesiar, E. Pap, Aggregation Functions, Encyclopedia of Mathematics and Its Applications, vol. 127, Cambridge University Press, Cambridge, 2009.

[7] M. Hermann, On Boolean primitive positive clones, Discrete Math. 308 (2008), 3151-3162.

[8] K. A. Kearnes, A quasi-affine representation, Internat. J. Algebra Comput. 5 (1995), 673702 .

[9] K. A. Kearnes, Á. Szendrei, The classification of commutative minimal clones, Discuss. Math. Algebra Stochastic Methods 19 (1999), 147-178.

[10] A. V. Kuznecov, On detecting non-deducibility and non-expressibility, Logical deduction, Nauka, Moscow, 1979, pp. 5-33 (in Russian).

[11] B. Larose, On the centralizer of the join operation of a finite lattice, Algebra Universalis 34 (1995), 304-313.

[12] E. L. Post, The Two-Valued Iterative Systems of Mathematical Logic, Annals of Mathematics Studies, no. 5, Princeton University Press, Princeton, 1941.

[13] A. Romanowska, J. D. Smith, Modes, World Scientific, Singapore, 2002. 
[14] V. Trnková, J. Sichler, All clones are centralizer clones, Algebra Universalis 61 (2009), no. $1,77-95$

[15] J. W. Snow, Primitive positive clones of groupoids, Algebra Universalis 60 (2009), no. 2, $231-237$.

[16] L. Szabó, On algebras with primitive positive clones, Acta Sci. Math. (Szeged) 73 (2007), no. $3-4,463-470$.

(M. Behrisch) Institute of Algebra, Dresden University of Technology, 01062 DresDen, Germany

E-mail address: mike.behrisch@mailbox.tu-dresden.de

(M. Couceiro) Mathematics Research Unit, University of Luxembourg, 6, Rue Richard Coudenhove-Kalergi, L-1359 Luxembourg, Luxembourg

E-mail address: miguel.couceiro@uni.lu

(K. A. Kearnes) Department of Mathematics, University of Colorado at Boulder, Campus Box 395, Boulder, Colorado 80309-0395, USA

E-mail address: kearnes@euclid.colorado.edu

(E. Lehtonen) Computer Science and Communications Research Unit, University of Luxembourg, 6, Rue Richard Coudenhove-Kalergi, L-1359 Luxembourg, Luxembourg E-mail address: erkko.lehtonen@uni.lu

(Á. Szendrei) Department of Mathematics, University of Colorado at Boulder, CamPus Box 395, Boulder, Colorado 80309-0395, USA

E-mail address: szendrei@euclid.colorado.edu 